ISSN 2602-8506

Vol. 2 / No.4/pág.50-67. Octubre-Diciembre/2018.

\title{
Herramientas de calidad total y la competitividad: caso sector carrocero de la provincia de Tungurahua.
}

Tools of total quality and competitiveness: Do you want to insure your car in the sector case province of Tungurahua.

Mario Fernando Navarrete Fonseca, Mg. ${ }^{1}$ Evelin Silvana Pilamunga Yanzapanta. ${ }^{2}$ Néstor Santiago Silva Ramos. ${ }^{3}$

\section{DOI: https://doi.org/10.33262/visionariodigital.v2i4.99}

\section{Resumen.}

La procuración de las empresas carroceras en el Ecuador en cuanto al manejo de herramientas de calidad es latente, pues del uso de ellas depende mucho si son competitivas en el mercado local e internacional. Lo que se busco es indagar de qué manera las herramientas de calidad total se relacionan con la medición de la competitividad productiva en el sector carrocero de la provincia de Tungurahua. El estudio tuvo métodos teóricos y empíricos, el enfoque fue cuantitativo. El diseño exploratorio, descriptivo y correlacional; la población de estudio 112 persona 96 clientes internos. La técnica para la recolección de datos la encuesta cuyo instrumento (cuestionario) estaba constituido por 23 ítems. Los resultados de mayor impacto fueron: que las herramientas de calidad total se relacionan con la competitividad productiva en el sector carrocero de la provincia de Tungurahua con un promedio de 0,850 , solo el $24 \%$ de las empresas cuentan con la certificación ISO, de la cantidad total de empresas carroceras Tungurahua ocupa un 65\% de participación, para contratar al personal no son tomadas en cuenta las competencias necesarias y no existe programas de reducción de costos en la producción. Fue conveniente elaborar la metodología para aplicación de herramientas de calidad.

Palabras clave: Metalmecánica, carrocerías, herramientas de calidad, calidad, competitividad y trabajo en equipo.

\footnotetext{
${ }^{1}$ Universidad Técnica de Ambato, Ambato, Ecuador (navarretemario1988@gmail.com)

${ }^{2}$ Universidad Técnica de Ambato, Ambato, Ecuador (silvana_2532@ @otmail.com)

${ }^{3}$ Universidad Técnica de Ambato, Ambato, Ecuador (santysr@yahoo.com) 


\section{Abstract.}

The management of companies which manufactured bodywork in Ecuador related to quality tools is required due to the use of them depends absolutely if they are competitive in the local and international market. It was researched how the total quality tools are related to the measurement of productive competitiveness in the bodywork sector of Tungurahua province. The study had theoretical and empirical methods, the approach was quantitative. The design was exploratory, descriptive and correlational; the study population was 112 people and 96 internal clients. The data collection technique was the survey which instrument was the (questionnaire) made of 23 items. Beyond the results the greatest impact we noticed was that the total quality tools are related to the productive competitiveness in the bodywork sector in Tungurahua province, reaching an average of 0.850 , just $24 \%$ of the companies have obtained the ISO certification, from the total of Bodyworks companies in Tungurahua it reaches $65 \%$ in the country. To hire employers, there are not taken into account the needed competences. In addition, there are no programs to reduce costs in production. It was appropriated to elaborate the Methodology for the application of quality tools in order to improve the competitiveness of the Tungurahua bodywork companies.

Keywords: Metalworking, bodywork, quality tools, quality, competitiveness, teamwork.

\section{Introducción.}

En la época actual la industria metalmecánica representa cerca de 16\% del PIB industrial en América Latina, ofrece empleo a 4.1 millones de personas en forma directa y 19.7 millones de forma indirecta. Tiene además una importante participación en el total de las exportaciones realizadas en la región. Para Alcántara (2015), en la Revista Metalmecánica Internacional tomada de su sitio web expone un listado por países de la participación que dicho sector mantiene, en Argentina representó 17.0\% del valor bruto de la producción en 2013; en Brasil fue 27.0\% del valor agregado manufacturero en 2012; para Colombia significó $10.4 \%$ del valor agregado en el sector manufacturero en 2012; y en México fue $31.0 \%$ del valor agregado manufacturero en 2012, de acuerdo con datos de la Asociación Latinoamericana del Acero.

Sin embargo, en los últimos tiempos, América Latina se ha tenido que enfrentar a dos retos importantes en su proceso de desarrollo económico: por un lado, la apertura de sus mercados y, por otro, la intervención de China en la competencia global, lo que ha llevado a un proceso de desindustrialización. En otras palabras, aunque muy afectada negativamente por las importaciones y la consiguiente eliminación de puestos de trabajo de alta calidad, la cadena de metalmecánica sigue siendo una de las actividades manufactureras más importantes de América Latina”. 
El Ecuador es muy conocido en el mundo por la exportación de varios productos como el banano, flores, etc.; por otro lado, hay muchos sectores económicos en el país que en la época actual han ido tomando fuerza, debido al cambio de la matriz productiva (Secretaría Nacional de Planificación y Desarrollo, 2012) y esto como tal se ha visto reflejado en los diferentes sectores económicos. La metalmecánica es el sector conformado por industrias relacionadas a la importación, producción, postproducción, exportación, entre otras, de productos de metal. Dentro de ésta, la construcción de carrocerías está tomada como una parte de la mecánica que se dedica a la producción de todo tipo de estructuras que puedan ser utilizadas para la fabricación de buses o medios de transporte urbano, rural, nacional o internacional.

En lo que se refiere a competitividad, según el enfoque antiguo, se entiende que la competitividad se encuentra referida a la medida en la que los precios de los bienes y servicios de un país pueden ser establecidos para poder competir con los de otros países. En palabras de Porter (1980), si se toma en cuenta que una de las principales metas económicas de un país es producir un alto nivel de vida para sus ciudadanos, la competitividad se encontraría vinculada a la capacidad de conseguir el bienestar, por lo que se encuentra determinada por el nivel de productividad con la que una nación, región o clúster, utiliza sus recursos naturales, humanos y de capital. Es entonces, que se puede decir que, el bienestar de un país se determina por medio de la productividad del mismo, alentándolo así a ser competitivo.

En todo el país se producen 127 carrocerías al mes, lo que suma 1.524 unidades al año. De este total, el 65\% sale de empresas de Tungurahua. Según un estudio de la CANFAC en todo el país cada año se renuevan cerca de 2.200 unidades de transporte de pasajeros. Se estima que al menos el $90 \%$ del nuevo parque automotor tiene carrocerías ensambladas en talleres nacionales. La calidad total es un factor predominante para el desarrollo de las empresas, en la provincia de Tungurahua concretamente en el sector carrocero (CANFAC), se agrupan 54 empresas que producen un estimado de 195 carrocerías mensuales, esto conlleva a la generación de competencias y la búsqueda de herramientas administrativas que permitan mejorar significativamente los procesos de calidad en de cada empresa carrocera. (Bombón, Vayas , Villacis , \& Gutierrez , 2017). Es así que el $65 \%$ de la producción nacional de carrocerías se centralizan en Tungurahua, estableciéndose como un sector productivo importante. El crecimiento exponencial de acuerdo a datos del Ministerio Coordinador de la Producción, Empleo y Competitividad indica que en 1990 existían 27 empresas en el sector carrocero del país y para el año 2005 se evidencia 180 empresas. (Fiallos, 2014).

En el Censo Nacional Económico del 2010 se detectó 183 empresas carroceras a Nivel Nacional 32 localizadas en la ciudad de Ambato (INEC, 2010). De esta manera se ha logrado estimar la dinámica económica de varios sectores como son el hierro y acero que tienen una participación del $34.5 \%$, en relación a la materia prima como la pintura y 
vidrio, con un $7.7 \%$, clavos, pernos y tornillos, $4.2 \%$ de aluminio y un $6.3 \%$ según (Fiallos, 2014). Es así que los nuevos requerimientos tecnológicos y de producción han llevado a las carrocerías a tomar nuevos estándares de calidad y certificación de sus procesos. De acuerdo a CANFAC (2015) el 24\% de las empresas tienen certificación de calidad ISO 9001, el 7\% en proceso de obtención de calidad y el $69 \%$ aun no incurren en este territorio (CANFAC, 2015).

La investigación conto con dos variables: variable independiente (herramientas de calidad) cuyas sub variables fueron: la alta gerencia, prevención, procesos, trabajo en equipo y programas de mejora y la variable dependiente (competitividad) las sub variables: toma de decisiones, planificación y factores (Gándara, 2014).

La base para fomentar el desarrollo y sistemas de evaluación de la calidad en el servicio según estudios realizados se remonta por el siglo XIX. En donde se comenzó a sintetizar la evaluación de la calidad, lo cual contribuyo en la conformación de estructuras, proceso y resultados en cuanto a tendencias de calidad. En ese entonces era muy complicado el definir la calidad por diferentes factores que se encontraban ligados a las diferentes actividades laborales que han dado un concepto de forma local. En 1999, el sistema de salud de los Estados Unidos genero una propuesta para la evaluación del desempeño, en donde se señalaba la gran necesidad de mejorar la atención adecuada, eficiencia y cuidado. Las empresas de actividades de servicios, consideran la necesidad de incrementar formas para mejorar la calidad del servicio (Fuentes, López, Rodríguez, \& López, 2013).

Mientras la demanda va incrementando con el pasar de los días, nace la gran necesidad de buscar soluciones dirigidas entre ellas está la construcción de modelos de calidad que se adopten a las diferentes actividades económicas. Con ello surgen además programas de evaluación y mejorara para las empresas, dando apertura para que se establezcan sistemas de evaluación y acreditación de las instituciones públicas y privadas. Por otra parte, se implementó proyectos de generación de herramientas de calidad en países de Europa y América Latina, desarrollando metodologías y criterios para la aplicación de cada una de ellas para el mejoramiento de los procesos de fabricación de los productos (Vigo , Segrea , León , \& López , 2014).

La búsqueda de disminuir los desaciertos en las actividades económicas a promovido que se deban identificar y diseñar herramientas de calidad, es así que se defina al as herramientas de calidad como estrategias para disminuir problemas internos y dar solución por medio de la aplicación y evaluación de las mismas. El utilizar herramientas de calidad es ser más eficientes y eficaces al tomar acciones para mejorar o disminuir le problema detectado, con lo cual las empresas pueden ser más competitivos (Gandara, 2014). 
Han trascurrido muchos años, es decir un milenio en la competitividad entre empresas que se ha vuelto una competencia más de permanencia que resistencia, por lo cual el rediseño de las empresas es fundamental y de mucha importancia para diferenciarse de la competencia. Pero esto se ha estado incrementado por la globalización de todos los mercados del mundo, mediante la diversidad de productos existentes y espacialmente en lo concerniente al objetivo de fortalecer el desarrollo. Por otro lado, crecían las polémicas por la dificultad de ubicar entre los objetivos de las políticas para incrementar la competitividad y la descentralización, por lo cual se desglosa un abanico de problemas y caminos que se encuentran constantemente en debate en las diferentes empresas (Morales, 2014).

Posterior al transcurrir los años el interpretar el término de competitividad se pudo realizar un análisis de la internacionalización, se puedo apreciar múltiples significados y alcances y ello se dio un enfoque macroeconómico y microecómico. Para controlar estas condiciones facilitan el entender la competencia desde un determinado entorno, el entender la macroeconomía conlleva a tener una mayor aproximación a la complejidad. Es por lo cual se considera a la competitividad con la capacidad que tiene una empresa o institución de mantener o generar ventajas que permitan mantenerse en el mercado y en el entorno que se rodeen. Por tanto, para que exista competitividad se debe generar herramientas que permitan evaluar el funcionamiento de las empresas, de allí la importancia de generar políticas e instrumentos de calidad (Botero, 2014).

Un estudio realizado en España revela que las herramientas de calidad contribuyen para la generación de competitividad en las empresas, debido que contribuye en la generación de políticas de desarrollo institucional en las diferentes arias y secciones de la empresa. La única forma de generar competitividad es generando el mismo que debe estar ligado a las actitudes y habilidades de la gerencia y departamentos (Molina, 2015).

El concepto de calidad tiene diferentes perspectivas y se diferencia de la actividad económica que se realiza la organización, pudriendo evidenciarse en el producto, servicio, proceso y producción de un bien (Carro Paz \& Gozález Gómez, 2012). Es por ello que la calidad es considerada como un conjunto de atributos y actividades con las que se pretenden asegurar que los productos cumplan con las características óptimas para la satisfacción de los clientes. Por otra la calidad es considerado como el conjunto de rasgos de un producto o servicios que se deben sustentar para obtener una habilidad para satisfacer las necesidades de los clientes (Carro Paz \& Gozález Gómez, 2012).

Existen varios gurús de la calidad debido a la variedad de estudios en referencia a la calidad total, principalmente por William Edwards Deming quien dedico casi cerca de treinta años para definir el ciclo PHVA (Planear, Hacer, Verificar y Actuar) que también es conocido como el Círculo de Deming (Constanza Cubillos \& Rozo Rodriguez, 2011).Por otra parte, Joseph Juran tubo un aporte significativo con la trilogía de Juran 
los cuales constan: Planeación de la calidad, Control de la calidad y la Mejora de la Calidad. Por su parte Kaoru Ishikawa por el año de 1960 estableció estándares de deben tener los productos o servicios lo cual se denominó como las ISO (International Standard Organización). Sus principales aportes fueron "sus siete herramientas básicas que son la Gráfica de Pareto, diagrama de causa efecto, estratificación, hoja de verificación, histograma, diagrama de dispersión y grafica de control de Stewart. (Constanza Cubillos \& Rozo Rodriguez, 2011).

Las herramientas de calidad son metodología que permiten gestionar la calidad de una organización, para que sean eficaces y eficientes cuando se pueda identificar un problema. Por tanto, es muy importante el identificar claramente el problema para poder determinar la herramienta de calidad más eficaz (Diagrama de Pareto, Ishikawa, Gantt, Deming, etc.) por los equipos de trabajo (Gándara, 2014).

El diagrama de Pareto según investigaciones realizadas se da el nombre en honor al economista y sociólogo Italiano Wilfredo Pareto (1848-1923), que fue ingeniero del instituto Politécnico de Turín. El origen del concepto de esta herramienta se remonta por el siglo XIX cuando Pareto determino que el $80 \%$ de la riqueza de Italia está en manos del $20 \%$ de la población, con lo cual se puedo evidenciar la generación de la teoría y los factores que se debían considerar. Por los años 50 Juran un tratadista de ese tiempo considerado que las observaciones de Pareto eran principios universales (Gándara, 2014).Con lo cual agrupo los factores que contribuyen a un efecto en común, para el tratamiento de la teoría en el desarrollo de las actividades buscando cuáles de ellos son los de mayor responsabilidad en cuanto al efecto. Es así que se define al diagrama de Pareto como una representación gráfica que tiene un sentido de orientación y guía en cuanto a su importancia o magnitud, fijando la frecuencia de ocurrencia de las distintas causas de un problema (Gándara, 2014).

Figura 1. Diagramas de Pareto

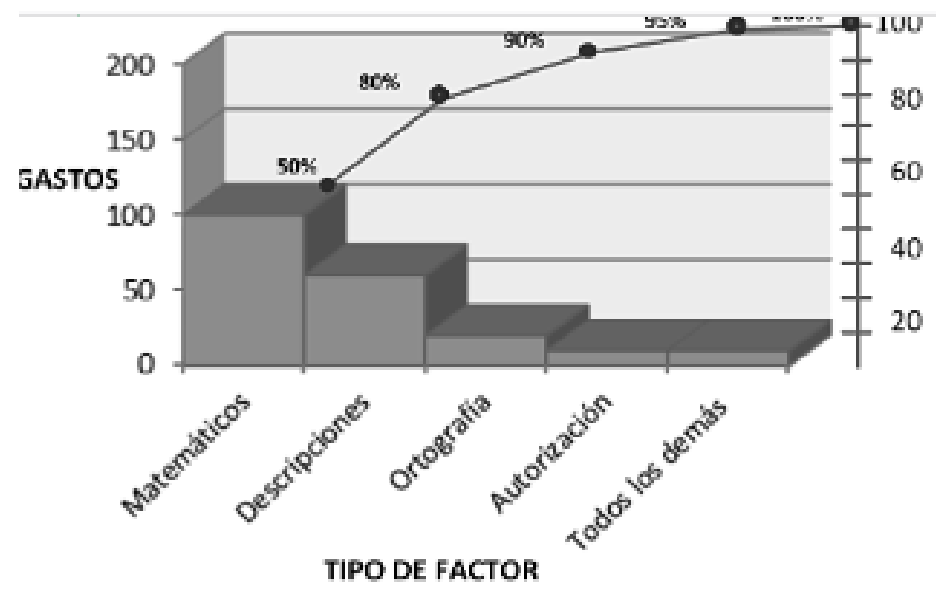

Elaborado por: Gándara, 2014. 
El diagrama de causa y efecto es considerado como el grado de dispersión de una variable en relación a la variación de otra que se debe controlar y tratar de reducir al mínimo posible. Esta herramienta lo que tiene como objetivo es evitar el riesgo de producir partes inadecuadas para su uso. Además, lo que se pretende es definir los límites de tolerancia especificados, con la finalidad de tener presente es buscar y mejorar la calidad del producto con la finalidad se satisfacer las necesidades del cliente. Al referimos a la variabilidad del diagrama se debe tener presente los factores como: materias primas, maquinaria o equipo, métodos de trabajo, mano de obra y le medio ambiente (Gándara, 2014).

Por tales aspectos o motivos puede existir una dispersión en la realización del proceso de producción, es por ello que puede existir una alta variabilidad en cuanto a la calidad. Además, es considerada como una técnica de análisis para buscar alternativas para dar solución a un problema detectado a ello se llama el diagrama de causa efecto, conocido también como Diagrama de Ishikawa, con el cual se puede analizar los factores que intervienen en la generación de la calidad de un producto identificado las relaciones existentes entre cada causa y efecto que da origen al problema, tomando en cuenta la forma de organizarse la información y la relaciones de mayor importancia. El diagrama de causa efecto se simboliza o recibe el nombre de "esqueleto de pescado", identificando que el dorsal de la espina denota el camino que se conduce a la cabeza del pescado en donde por lo general se coloca el problema identificado, posterior se debe analizar las espinas que gráficamente son en forma de flechas que indican las causas y sub causas lo cual se evidencia en la siguiente ilustración.

Figura 2. Diagrama de causa y efecto.

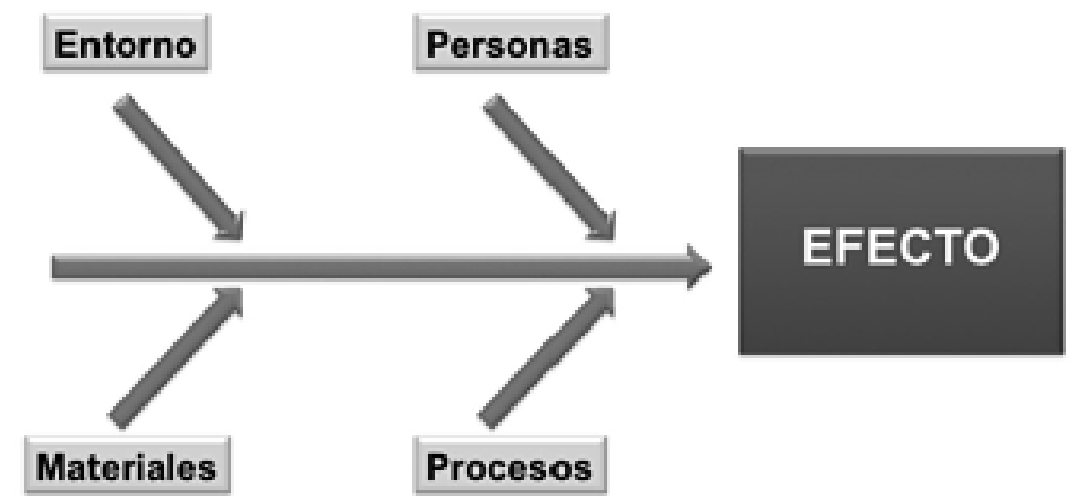

Elaborado por: Gándara, 2014.

El modelo de mejora continua e basa en la idea fundamental al referirse a los procesos implicados en el aspecto de servicios de asistencia y tratamiento que son tareas separadas, con lo cual se identifica las actividades que generan las funciones. El círculo de Deming 
es también conocido como el ciclo de PDCA por sus siglas en inglés, el mimo que consiste en el conjunto de cuatro elementos que son tratados consecutivamente. Estos elementos son: P: Planear: establecer los planes, D: Hacer: llevar a cabo los planes, C: Verificar: verificar si los resultados concuerdan con lo planeado, A: Actuar: actuar para corregir los problemas (Gándara, 2014).

Figura 3. Circulo de Deming.

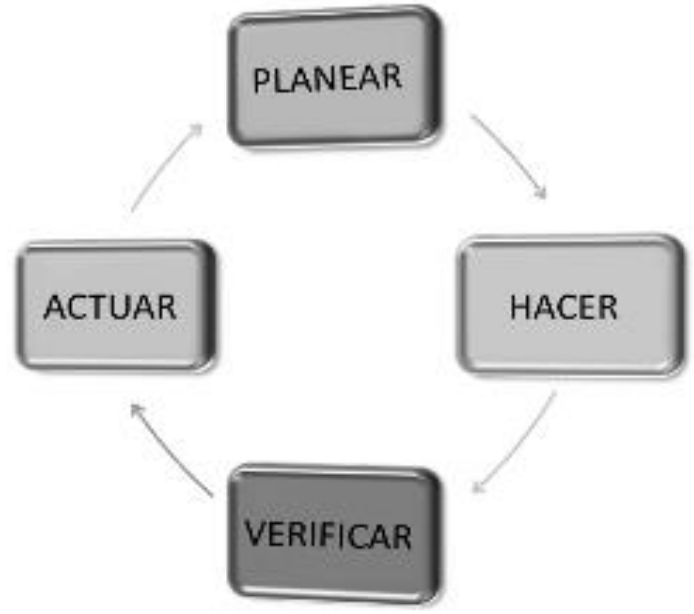

Elaborado por: Gándara, 2014.

La competitividad es considerada como un tema de mucha relevancia en las diferentes organizaciones, pues por ello se comprometen de mayor manera y se relacionan con sus clientes y la referencia de un producto o servicio. Por tanto, es necesario el comprender que la competitividad va acompañada por una innovación en los niveles de calidad y competitividad. Además, la competitividad está estrechamente relacionada con las herramientas de calidad y es parte fundamental para encaminar adecuadamente (Pinzón, 2014). En otras palabras, la competitividad es la capacidad que tienen las empresas de generar ventajas que les permitan superar a sus competidores locales e incursionar en nuevos mercados, mejorando de esta forma la calidad de vida de la población. Por consiguiente, puede considerarse a la competitividad como parte importante del entorno empresarial se relacionada con el incremento de los niveles de eficiencia y eficacia interna de la organización.

En el mundo empresarial y de mercadeo la importancia de la competitividad radica en las ventajas que tener un buen nivel de competitividad como medio para lograr que las organizaciones más eficientes al utilizar y manejar los recursos. Se debe recalcar que la competitividad no surge de la espontaneidad sino de un proceso muy ampliado tanto de aprendizaje como negociación. La competitividad no es producto de una casualidad ni surge espontáneamente, se crea y se logra a través de un largo proceso de aprendizaje y negociación. Por lo tanto, para un correcto desarrollo competitivo empresarial, es 
necesaria la aplicación de estrategias que involucren la participación de directivos, empleados, proveedores, clientes y, por último, del gobierno y la sociedad en general. (Chisco, 2013).

El concepto de competitividad se puede abordar de diferentes niveles teniendo en cuenta el entorno que la rodea la empresa para identificar a las empresas competidoras para identificar si es competitiva. Entre los factores de mayor influencia en la competitividad está el territorio y agentes económicos; en el plano de los agentes económicos está relacionado o su impacto está relacionado con el comercio internacional enfocado a los enfoques de ventajas absolutas y ventajas comparativas y ello varia del consumo de bienes si existen intercambios en el marco económico (Bernal \& Mungray, 2017).

Sin duda a lo anterior mencionado para ser competitivos, se debe apostar al conocimiento y al uso de nuevas herramientas tecnológicas y de calidad, con lo cual se puede asegurar la capacidad para generar cambios de mucha jerarquía y de decisión para enfrentar la evolución del mercado (Molina, 2015). Por tanto, se define a la competitividad como la capacidad que tiene ciertas empresas públicas o privadas dependiendo de su actividad económica, ello debe ser de forma sincronizada, sistemáticamente ordenada con la finalidad de generar ventajas representativas para poder mejorar la posición en el nicho de mercado que pertenezca en un entorno socioeconómico. Toda ventaja está dada por el conjunto de competencias que tengan el talento humano, los recursos, conocimientos de parte de la gerencia con lo cual se puede competir con las demás empresas (Botero, 2014). La toma de decisiones tiene varias perspectivas, pues lo que se pretende al tomar una decisión es alcanzar sus propios fines en relación a los problemas o necesidades existentes en una empresa, pero al existir varias alternativas para decidir se debe identificar las ventajas positivas y desventajas que se pueden adquirir al tomar la decisión. Por lo cual los administradores sugieren el emplear modelos matemáticos y estadísticos para estimar una alternativa que permita disminuir el riesgo al decidir siempre y cuando no estén afectando con los objetivos institucionales (Espinosa, 2016).

La toma de decisiones es considerada con un proceso de carácter intelectual que facilita una elección entre diferentes posibilidades que tengan semejanza en varias situaciones. Es por ello que también se considera con un proceso opcional para dar solución a problemas de un individuo de organización, el cual debe ser continuo y ello podrá variar dependiendo de los objetivos que se plantean y los recursos que se disponga al elegir una alternativa (Moncada \& Sofía, 2013).

\section{Métodos y materiales.}

El desarrollo de la investigación se dio por medio de métodos teóricos como: histórico lógico, inductivo-deductivo y analítico sintético El estudio se realizó con el apoyo de métodos teóricos histórico lógico, inductivo-deductivo y analítico sintético con los cual se construyó el sustento teórico (Del Cid, Méndez, \& Sandoval, 2011). Además, el 
método empírico que tuvo el apoyo de las formas de recopilación de datos (Hernández, Fernández, \& Baptista, 2010).Entre las formas de recopilación estuvieron datos primarios y secundarios. El enfoque fue cuantitativo debido a la forma estructurada que se realizó la investigación y también porque se comprobó las hipótesis y se generó resultados (Becerra, 2016).

El diseño de la investigación en general es la estrategia que se plantea con la finalidad que responda a la necesidad del problema planteado. Por ello el estudio se partió de una investigación exploratoria en vista que no se contaba con un modelo o estructura preestablecida para direccionar la investigación, el contribuyo para entender y comprender el problema de estudio (Fidias, 2012). Posterior una vez que se comprendió el problema se enrumbo a un nivel descriptivo con el cual se pudo identificar las causas, efectos y características que dieron origen al problema. Pero el estudio como se trató a mayor profundidad se recurrió a un nivel correlacional el cual facilito en comprender las relaciones que existían entre ambas variables, para ello se plantío hipótesis que fueron comprobadas por el modelo de (Coeficiente de Pearson), el cual admite relaciones tanto positivas y negativas (Fernández, Hernández, \& Baptista, 2010).

Para el desarrollo del estudio se utilizó la técnica de la encuesta cuyo instrumento fue le cuestionario estructurado, el mismo que estuvo compuesto por 23 ítems de los cuales tres ítems eran de datos generales, 18 de escala de Likert y 2 de opciones múltiples. Para las preguntas de escala de Likert los extremos fueron "Totalmente en desacuerdo" y "Totalmente de acuerdo". Los cuales fueron fraseados al momento que se revisión la literatura al construir el marco teórico. El instrumento tuvo dos aplicaciones la primera que de forma piloto en donde se validó la consciencia de los ítems del instrumento y se realizó al $10 \%$ de la población de estudio y la segunda aplicación que fue a toda la población es decir al gerente y jefe de producción de las 56 empresas carroceras, con lo cual se pudo comprobar las hipótesis y redactar los resultados (Fernández, Hernández, \& Baptista, 2010).

La se entiende como la cantidad total de elementos o individuos que interactuarán en el estudio y se aplicara algún instrumento para levantar la información. Para este caso la población de estudio estará compuesta por 112 personas que pertenecen a las a las 56 empresas carroceras registradas den la CANFAC de la provincia de Tungurahua como se muestra en la siguiente Tabla 1 (Fidias, 2012).

La validación del instrumento está dada por dos parámetros: el primero es que si en verdad el instrumento mide lo que se quiere. Por ello se consideró realizar la validez por expertos el cual consistió en presentar el instrumento a 4 docentes de la Facultad de Ciencias Administrativas con lo cual se procedió aprobar y realizar cambios. Por otra parte, el factor la confiabilidad es el número de vez que se aplicó el instrumento en investigaciones parecidas de acuerdo con Fernández, Hernández, \& Baptista (2010), los cuales mencionan 
que la confiabilidad esta dado también por el número de ítems compuestos. El instrumento estuvo compuesto por dos partes la primera datos informativos y la segunda el desarrollo de las dimensiones de cada variable de estudio.

El proceso de confiabilidad se realizó aplicando el instrumento de forma piloto al $10 \%$ de la población total. Lo primero que se hizo es ingresar los valores obtenidos en el software SPSS Statistics versión 23.0. Una vez ingresados se tomó la opción de análisis de fiabilidad (índice de Alfa de Cronbach), pus el índice permite entender si existe consistencia interna entre ítems (Celina \& Campo, 2005).El criterio para decidir es que el valor arrojado por el software debe ser superior al 0,70 que indica que si existe consistencia entre los ítems(Quero, 2010).

Tabla 1. Estadísticas de fiabilidad del instrumento de medición estadística

\begin{tabular}{ll}
\hline Alfa de Cronbach & N de elementos \\
\hline 964 & 23 \\
\hline
\end{tabular}

Elaborado por: Grupo de Investigadores

Por otra parte, el estudio conto con dos hipótesis (Ho) Nula: "Herramientas de calidad total no se relacionan con la competitividad productiva en el sector carrocero de la provincia de Tungurahua" y (Ha) Alterna: "Herramientas de calidad total se relacionan con la competitividad productiva en el sector carrocero de la provincia de Tungurahua", las mismas las cuales fueron verificadas a través del modelo estadístico (Coeficiente de correlación de Pearson), el mismo que permite identificar las relaciones entre los diferentes componentes de cada variable de estudio. Cabe mencionar que las relaciones pueden ser positivas o negativas y no busca la causalidad. (Arriaza, s/n). Además, el procesamiento de la información y modelo fue aplicado en el software SPSS Statistics versión 23.0.

\section{Análisis e interpretación de los resultados.}

Es así que se puede evidenciar que la mayor parte de los encuestados consideran que la alta gerencia participa activamente en la gestión de la calidad. Pues la alta gerencia es conocida como alta dirección en el aspecto corporativo la misma que cumple tres tipos de funciones corporativas, entre las principales actividades están: definir el portafolio, gestión interna y gestión externa. Al referimos por la definición del portafolio que consiste e identificar el conjunto de negocios, es decir se centra en una serie de preguntas las cuales deben ser contestadas (Rivas, 2015).

La mayor parte de los encuestados desconocen que la empresa evalúa las políticas y planes de calidad. Pues se debe seleccionar un sistema de evaluación en los procesos es una tarea muy complicada debido que deben identificar las ventajas y desventajas que se 
pueden percibir. Pues es considerada la evaluación con el proceso por él se compara los objetivos planteados con el cumplimiento de los mismos (Capó, Oliver, \& Sard, 2013). Los encuestados consideran que desconocen si la empresa cuenta con procesos de control o ha vez no se los aplica al momento de realizar los procesos productivos. Esto debido que las empresas buscan optimizar el sistema de procesos vinculado a generar satisfacción los clientes y a las demás personas de interés o que rodena a la empresa y a sus actividades empresariales (LLanes, Cira, Moreno, \& García, 2014).

Los encuestados consideran que ha existido mejoramiento de los procesos para fabricar los diversos productos carroceros. Ello debido que El mejoramiento de procesos son acciones que considera una organización, para ello se debe revisar adecuadamente el cumplimento de requerimientos necesarios. Además, se debe considerar las nuevas incorporaciones y renovaciones de acuerdo con los antecedentes que tenga, la práctica diaria ha demostrado es casi imposible mantener políticas estáticas para generar calidad en los productos (Cajas, 2013).

Los encuestados desconocen si los trabajadores cuentan con capacitaciones específicas para cada puesto de producción. Pues la capacitación según varios estudios realizados el actualizar los conocimientos en la práctica profesional que es una característica de mucho interés e importancia. Además, de la actualización de conocimientos no se debe descuidar el intercambio de experiencias entre los participantes, con ello se puede compartir soluciones (Fuentes, 2013). Para la comprobación de la hipótesis se empleó el modelo estadístico (Coeficiente de correlación de Pearson), el mismo que permite identificar las relaciones entre los diferentes componentes de cada variable de estudio. Cabe mencionar que las relaciones pueden ser positivas o negativas y no busca la causalidad.

Tabla 2. Correlaciones significativas.

\begin{tabular}{|c|c|c|c|c|c|c|c|c|c|}
\hline & & Cumplimiento & Procesos & Entrenamiento & Comisión & Competencias & Reducción & Innovación & Objetivos \\
\hline \multirow{3}{*}{ Cumplimiento } & $\begin{array}{l}\text { C. } \\
\text { Pearson }\end{array}$ & 1 &, $888^{* *}$ &, $881^{* *}$ &, $903^{* *}$ &, $836^{* *}$ &, $842^{* *}$ &, $836^{* *}$ &, $842^{* *}$ \\
\hline & Sig. & & 0 & 0 & 0 & 0 & 0 & 0 & 0 \\
\hline & $\mathrm{N}$ & 112 & 112 & 112 & 112 & 112 & 112 & 112 & 112 \\
\hline \multirow{3}{*}{ Procesos } & $\begin{array}{l}\text { C. } \\
\text { Pearson }\end{array}$ & & 1 &, $912^{* *}$ &, $938^{* * *}$ &, $879^{* *}$ &, $894^{* *}$ &, $895^{* *}$ &, $893^{* *}$ \\
\hline & Sig. & & & 0 & 0 & 0 & 0 & 0 & 0 \\
\hline & $\mathrm{N}$ & & 112 & 112 & 112 & 112 & 112 & 112 & 112 \\
\hline \multirow{3}{*}{ Entrenamiento } & $\begin{array}{l}\text { C. } \\
\text { Pearson }\end{array}$ & & & 1 &, $901^{* *}$ &, $925^{* *}$ &, $926^{* *}$ &, $893^{* *}$ &, $860^{* *}$ \\
\hline & Sig. & & & & 0 & 0 & 0 & 0 & 0 \\
\hline & $\mathrm{N}$ & & & 112 & 112 & 112 & 112 & 112 & 112 \\
\hline \multirow{3}{*}{ Comisión } & $\begin{array}{l}\text { C. } \\
\text { Pearson }\end{array}$ & & & & 1 &, $910^{* *}$ &, $863^{* *}$ &, $922^{* *}$ &, $930^{* *}$ \\
\hline & Sig. & & & & & 0 & 0 & 0 & 0 \\
\hline & $\mathrm{N}$ & & & & 112 & 112 & 112 & 112 & 112 \\
\hline Competencias & $\begin{array}{l}\text { C. } \\
\text { Pearson }\end{array}$ & & & & & 1 &, $828^{* *}$ &, $907^{* *}$ &, $921^{* *}$ \\
\hline
\end{tabular}


ISSN 2602-8506

WWW.visionariodigital.org

\begin{tabular}{|c|c|c|c|c|c|}
\hline & Sig. & & 0 & 0 & 0 \\
\hline & $\mathrm{N}$ & 112 & 112 & 112 & 112 \\
\hline \multirow{3}{*}{ Reducción } & $\begin{array}{l}\text { C. } \\
\text { Pearson }\end{array}$ & & 1 &, $872^{* *}$ & ,801** \\
\hline & Sig. & & & 0 & 0 \\
\hline & $\mathrm{N}$ & & 112 & 112 & 112 \\
\hline
\end{tabular}

Elaborado por: Grupo de Investigadores.

Se puede denotar que la correlación de mayor influencia de la primera fila es entre cumplimiento-comisión con un índice de $(0,903)$, con lo cual se demuestra que existe una correlación positiva media. Pues el cumplimiento de funciones al proceso de verificación del desarrollo de las actividades encomendadas a un puesto de trabajo en específico, y cuya verificación debe estar a cargo de la autoridad competente dependiendo el nivel que posea (Urdaneta, García, \& Maldonado, 2015).

Por otra parte, en la fila dos la correlación de mayor aporte fue entre procesos-comisión con un índice de $(0,938)$, con lo cual se demuestra que existe una correlación positiva media. Pues las empresas buscan optimizar el sistema de procesos vinculado a generar satisfacción los clientes y a las demás personas de interés o que rodena a la empresa y a sus actividades empresariales (LLanes, Cira, Moreno, \& García, 2014).

En la tercera fila de las correlaciones se evidencia que la relación de mayor relevancia fue entre los entrenamiento-competencias con un índice de $(0,926)$, con lo cual se muestra una correlación positiva media. Ello debido que las competencias son la combinación adecuada de conocimiento, habilidades, actitudes, predisposiciones que permiten la motivación y capacidades que direccionan a la actuación eficiente de diferentes tareas y actividades, cuyo fin es lograr los objetivos decíamos por la organización. Además, es considerado como una condición indispensable para el uso del conocimiento como estratégico (Cejudo, 2017).

En la cuarta fila de las correlaciones se demuestra que no es muy significativa entre el comisión-objetivos $(0,930)$, con lo cual se demuestra una correlación positiva considerable. Pues la comisión es un conjunto de personas que se reúnen mediante un determinado tiempo donde se deban y se revisan políticas para mejorar la calidad de los procesos y productos (Suarez, Solarte, \& Cuéltar, 2013).

En la quinta fila de las correlaciones se demuestra que no es muy significativa entre el competencias-objetivos $(0,921)$, con lo cual se demuestra una correlación positiva considerable. Las competencias son la combinación adecuada de conocimiento, habilidades, actitudes, predisposiciones que permiten la motivación y capacidades que direccionan a la actuación eficiente de diferentes tareas y actividades, cuyo fin es lograr los objetivos decíamos por la organización. Además, es considerado como una condición indispensable para el uso del conocimiento como estratégico (Cejudo, 2017). 
Al ser los valores calculados son más cercanos "0" igual a "1" se rechaza la hipótesis nula y se aprueba la alterna: "Herramientas de calidad total se relacionan con la competitividad productiva en el sector carrocero de la provincia de Tungurahua". Lo cual muestra la prueba estadística que existe una (correlación positiva considerable) (Visauta, 2007).

\section{Conclusiones.}

- Luego de hacer revisado cada una de las herramientas de calidad total se evidencia que sirven como un instrumento para medir la competitividad productiva y ello depende mucho del producto, servicio y procesos productivos que los realice. Pues las herramientas de calidad lo que buscan es garantizar el conjunto de actividades en la empresa y asegurar los productos que obtengan niveles óptimos de calidad con lo cual se puede medir la competitividad productiva de una empresa en comparación de otra.

- Al revidar la literatura y las diferentes teorías se pudo evidenciar que al transcurrir el tiempo las herramientas se han innovado para medir la competitividad y la eficiencia de las empresas. Entre las que más se destacan están: en cuanto a los programas de calidad se encuentran la mejora continua, círculo de la calidad, cero defectos y 6 sigma. Por otra parte, las herramientas son: Hoja de verificación, Gráfico de control de procesos, Método Taguchi, Método de Kano, Diagrama de flujo y Benchmarking.

- La situación actual de las empresas carroceras en la provincia de Tungurahua según la CANFAC en el país se produce 1524 unidades al año, de este total el $65 \%$ sale de las empresas de Tungurahua. Pero las importaciones de unidades por parte de las diferentes empresas son considerables, pues el país se renuevan cerca 2200 unidades lo cual encuentra afectando al sector carrocero local. En cuanto a la calidad total el 24\% de las empresas cuenta con la calidad ISO 9001, E L7\% están en proceso y el $69 \%$ aun no incurren en este territorio. Pero cabe mencionar que si utilizan herramientas de calidad para el control de la producción.

- Luego de haber analizado los componentes y herramientita de la calidad y competitividad se evidencia que las herramientas más utilizadas es el grafico de control de procesos y la hoja de verificación, los mismos que se emplean neta mente para controlar los procesos de producción, pero en algunos casos de las empresas encuestadas expresaron que utilizan otras herramientas que no tiene el sustento teórico y técnico para usarlas. En cuanto a la competitividad se desconoce que exista capacitación para los trabajadores, las competencias requeridas para contratar a los trabajadores en su mejorar no se cumplen, no existe programas para la reducción de costos en la producción.

\section{Referencias bibliográficas.}


- Becerra, G. (2016). Los usos del constructivismo en las publicaciones científicas de Latinoamérica. Mad. Revista del Magíster en Análisis Sistémico Aplicado a la Sociedad(35), 38-59. E-ISSN: 0718-0527

- Bernal, H., \& Mungray, A. (2017). Los índices de competitividad en México. Gestión y Política Pública, XXXVI(1), 167-218. ISSN: 1405-107

- Bombón, D., Vayas , G., Villacis , J., \& Gutierrez , E. (2017). Caracterización del entorno empresarial proveedor del sector carrocero de la provincia de Tungurahua. Congreso internacional Ciencia Sociedad e Investigación Universitaria. Ambato.

- Botero, L. (2014). Internacionalidad y Competiividad. Ciencias Estratégicas, XXII(32), 187-196. ISSN: 1794-8347

- Cajas, C. (2013). Mejoras en la gestión del proceso edit. Argentina de Radiolo, LXXVII(3), 193-194. ISSN: 0048-761

- Capó, J., Oliver, X., \& Sard, M. (2013). Evaluando la evaluación continua. @ tic. revista d'innovació educativa(10), 33-43. E-ISSN: 1989-3477

- Carro Paz, R., \& Gozález Gómez, D. (2012). Administracion de la Calidad Total. La Plata, Argentina.

- Celina, H., \& Campo, A. (2005). Aproximación al uso del coeficiente alfa de Cronbach. Colombiana de Psiquiatría, XXXIV(4), 572-580. ISSN: 0034-7450

- Chisco, N. (2013). La estrategia de diferenciación como herramienta competitiva para las pequeñas empresas sector de la confeccion. Mexico.

- Constanza Cubillos, M., \& Rozo Rodriguez, D. (2011). El Concepto de Calidad. Universidad de la Salle, 20.

- Espinosa, C. (2016). ¿Cómo se toman las decisiones organizacionales? Una revisión clásica. Sociológica, XXXI(87), 43-78. ISSN: 0187-0173

- Fernández, C., Hernández, R., \& Baptista, M. (2010). Metodología de la Investigación (Quinta ed.). Distrito Federal, México: Mc Graw-Hill. ISBN: 978607-15-0291-9

- Fiallos, J. G. (2014). Estudio de la cadena productiva del sector metalmecánico - carrocero de la provincia de tungurahua cantón ambato. Quito. Obtenido de http://repositorio.uta.edu.ec/bitstream/123456789/20619/1/T2626i.pdf 
- Fidias, G. (2012). El proyecto de investigación ( $6^{\circ}$ ed.). Caracas, Venezuela: Episteme. ISBN: 980-07-8529-9

- Fuentes, L. (2013). Capacitación económica sobre capital de trabajo. EduSol, XI(42), 56-64. E-ISSN: 1789-809

- Fuentes, Z., López, S., Rodríguez, O., \& López, S. (2013). Herramienta de calidad en el paciente propuesto para cirugía electiva no cardiaca. Archivo Médico de Camagüey, XVII(2), 162-173. E-ISSN: 1025-0255

- Gandara, F. (2014). Herramientas de calidad y el trabajo en equipo para disminuir la reprobación. Conciencia Tecnológica(48), 17-24. ISSN: 1405-5597

- Gándara, F. (2014). Herrmaientas de claidad y el trabajo en equipo para disminuir la reprobación escolar. Conciencia Tecnológica(48), 17-24. ISSN: 1405-5597

- LLanes, M., Cira, I., Moreno, M., \& García, G. (2014). De la gestión por procesos a la gestión integrada por procesos. Ingeniería Industrial, XXXV(3), 255-264. ISSN: 0258-5960

- Molina, N. (2015). Conocimiento, preámbulo de competiti. Revista Mexicana de Economía yFinanzas. Nueva Época / MexanJournal of Economics and Fin, X(2), 1-2. ISSN: $1665-534$

- Moncada, A., \& Sofía, M. (2013). Toma de decisiones clínicas en atención primaria. Médica Heredia, XXXIV(24), 319-323. ISSN: 1018-130X

- Morales, E. (2014). La dimensión territorial de la competitividad. Economía y Desarrollo, XVI(1), 71-84. ISSN: 0252-8584

- Pinzón, L. (2014). Internacionalización y Competitividad. Revista Ciencias Estrategicas, 22(32), 187-196.

- Quero, M. (2010). Confiabilidad y coeficiente Alpha de Cronbach. Telos, XII(2), 248-252. ISSN: $1317-0570$

- Rivas, L. (2015). El equipo de altagernecia en una mepresa multinegocios. Caso suramericana S.A. Ciencias Estratégicas, XXIII(23), 121-133..ISSN: 1794-8347

- Vigo , P., Segrea , J., León, B., \& López , T. (2014). Autoevaluación institucional. Una herramienta indispensable en la calidad de los procesos universitarios. MediSur, XII(5), 72-735. E-ISSN: 1727-897X 


\section{Para citar el artículo indexado.}

Navarrete M., Pilamunga E. \& Silva N. Herramientas de calidad total y la competitividad: caso sector carrocero de la provincia de Tungurahua. Revista electrónica Visionario Digital 1(1), 40-67. Recuperado desde: http://cienciadigital.org/revistacienciadigital2/index.php/VisionarioDigital/article/view/99/ $\underline{93}$

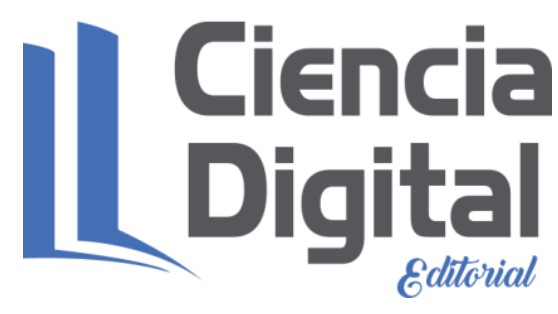

El artículo que se publica es de exclusiva responsabilidad de los autores y no necesariamente reflejan el pensamiento de la Revista Ciencia Digital.

El articulo queda en propiedad de la revista y, por tanto, su publicación parcial y/o total en otro medio tiene que ser autorizado por el director de la Revista Ciencia Digital.
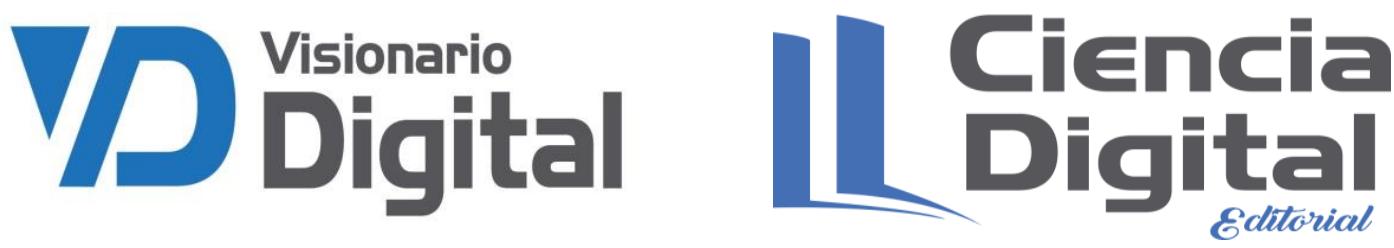